\title{
WEAK INFRARED MOLECULAR LINES REVEAL RAPID OUTFLOW IN COOL MAGNETIC SUNSPOT PENUMBRAL FIBRILS
}

\author{
M. J. Penn, ${ }^{1,2}$ W. D. Cao, ${ }^{3,4}$ S. R. Walton, ${ }^{2}$ G. A. Chapman, ${ }^{2}$ and W. Livingston ${ }^{1}$ \\ Received 2003 April 8; accepted 2003 May 8; published 2003 May 21
}

\begin{abstract}
New imaging spectropolarimetric observations of the Evershed flow in sunspot penumbrae using weak infrared molecular absorption lines are presented. A plane-polar coordinate system in the sunspot frame is defined, allowing averaging of many raw spectra. Molecular lines show Doppler shifts implying typical horizontal outflow speeds of 6 and up to $9 \mathrm{~km} \mathrm{~s}^{-1}$. The Ti I polarimetric spectra show the same rapid outflow and suggest an average penumbral magnetic field strength of $1400 \mathrm{G}$. While these observations show Doppler shifts of the entire line profile, the velocities are in better agreement with previous measurements from spectral line asymmetries.
\end{abstract}

Subject headings: Sun: atmospheric motions — Sun: infrared — sunspots — techniques: spectroscopic

\section{INTRODUCTION}

The penumbra of a sunspot marks the region where the strong vertical magnetic fields and cool plasma present in the sunspot umbra merge with the widely dispersed magnetic fields and hotter plasma of the quiet Sun. The penumbra is dominated by thin radial fibrils that appear either bright or dark. Recent imaging observations using adaptive optics show fibrils 150$180 \mathrm{~km}$ in width containing dark narrow cores less than $90 \mathrm{~km}$ wide (Scharmer et al. 2002). Since objects of $180 \mathrm{~km}$ on the Sun subtend angles smaller than 0 .'25 when viewed from the Earth, these penumbral fibrils provide challenging targets to image even at solar observatories with the best seeing conditions. Early spectroscopic observations by Evershed detected plasma flows crossing the penumbra directed radially outward from the sunspot (Evershed 1909), and these flows are named in his honor. The difficulty in obtaining spectral observations with the high spatial resolution needed to resolve the small penumbral fibrils has been the source of much confusion concerning the properties of the Evershed flow (Wiehr \& Degenhardt 1992). Although several empirical models of the gas properties in the penumbra have been developed (Moe \& Maltby 1974; Ding \& Fang 1989), a recent spectral line analysis has successfully used a simple linear combination of a quiet Sun model and a sunspot umbral model rather than a new penumbral model (Solanki, Montavon, \& Livingston 1994). The success of this modeling effort and recent observations of Evershed downflows in cold penumbral regions (del Toro Iniesta, Bellot Rubio, \& Collados 2001) suggests that spectral diagnostic techniques previously used to study the umbra of sunspots can be used to investigate the penumbral regions. In particular, spectral absorption lines from molecules have been used to diagnose plasma properties inside sunspot umbra (Bhatnagar, Livingston,

\footnotetext{
${ }^{1}$ National Solar Observatory (operated by the Association of Universities for Research in Astronomy, Inc., under cooperative agreement with the National Science Foundation), 950 North Cherry Avenue, Tucson, AZ 85719; mpenn@noao.edu.

${ }^{2}$ Department of Physics and Astronomy, San Fernando Observatory and California State University Northridge, 18111 Nordhoff Street, Northridge, CA 91330-8268.

${ }^{3}$ Yunnan Observatory, National Astronomical Observatories, Chinese Academy of Sciences, P.O. Box 110, 650011 Kunming, Yunnan, China.

${ }^{4}$ Center for Solar Research, New Jersey Institute of Technology, 323 Martin Luther King Boulevard, Newark, NJ 07102.
}

\& Harvey 1972; Penn \& LaBonte 1993), and these diagnostics are thought to be independent of telescopic scattered light and blurring caused by terrestrial atmospheric seeing, since the spectral lines are formed only at the cool temperature of the umbral plasma (see Fig. 1). Here new observations of Evershed flows using molecular lines are presented, and it is shown that these lines reveal rapid flows that must be present in the cool dark magnetic penumbral fibrils.

\section{OBSERVATIONS}

The Evershed flow has been proposed to be a flow along the mostly horizontal magnetic field lines in the penumbra (Beckers $\&$ Schroter 1969). Observations of sunspots near the center of the solar disk have measured the vertical components of the Evershed flow (Rimmele 1995b; Westendorp Plaza et al. 1997, 2001) and in some cases have detected supersonic downward motions (del Toro Iniesta et al. 2001). Most observations measure the horizontal component of the flow as seen when solar rotation carries sunspots near the limb of the Sun, and this work analyzes sunspots near the limb. From 2002 June 21 through 29, the sunspot NOAA Active Region 10008 was observed with the National Solar Observatory (NSO) McMath-Pierce (McM/P) solar telescope at Kitt Peak, Arizona, using the joint California State University Northridge-NSO infrared camera system. Spectral regions were observed at 1565 (June 21 through 27) and $2231 \mathrm{~nm}$ (June 29) containing lines of neutral iron and titanium (Fe I and Ti I) and several identified and unidentified molecular species. Two-dimensional scans of the solar surface, each containing more than 30,000 individual spectra, were taken of the sunspot on each day. The spatial resolution of the scans was $1^{\prime \prime} \times 1^{\prime \prime}$, and the sunspot was located from 0.27 to 0.91 solar radii from disk center; further details of these observations are presented elsewhere (Penn et al. 2003a, 2003b). Although the NSO McM/P is the world's largest solar telescope, the individual penumbral spectra are photon limited and the noise levels cause difficulties in measuring the molecular spectral lines. Azimuthal bins are used (see Fig. 2) to average between 300 and 600 individual spectra in order to reduce the photon noise below the level of the weak molecular lines; radial limits (roughly 1-5 times the umbral radius) and temperature limits (roughly 5400$6000 \mathrm{~K})$ were imposed to identify penumbral pixels. The resulting spectral plots for the $\mathrm{CN}$ absorption line at $1564.6 \mathrm{~nm}$ and for a weak unidentified molecular line at $2232.2 \mathrm{~nm}$ (which 


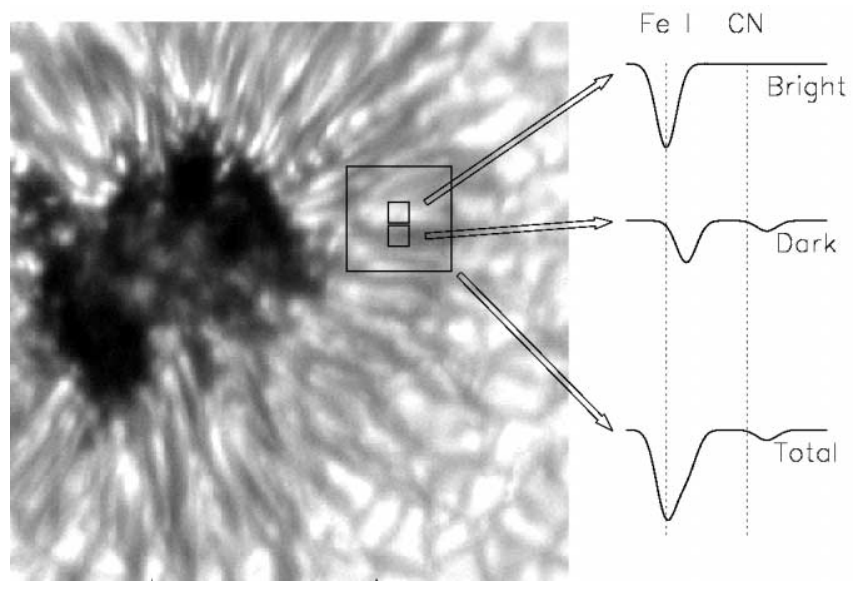

FIG. 1.-Image of a sunspot taken by Thomas Rimmele at the NSO Dunn Solar Telescope with high-order adaptive optics, showing penumbral filaments. The field of view is about $25^{\prime \prime}$ on each side (about $18,000 \mathrm{~km}$ on the Sun). A neutral atomic line such as Fe I can be observed in the bright filaments and also in the dark filaments; the simplifying assumption in this figure is that the dark filaments are the location of Evershed outflow and produce a redshift in the Fe I line. When scattered light, atmospheric seeing, or postprocessing add the spectra from dark and light filaments, an asymmetric $\mathrm{Fe}$ I line profile results. A molecular absorption line such as $\mathrm{CN}$ has a strong dependence on local plasma temperature and is not formed in the bright filaments, only in the cool dark filaments. The measured molecular line profile reveals the cool outflow as a Doppler shift of the entire line profile.

has a peak equivalent width at $4500 \mathrm{~K}$, similar to $\mathrm{CN}$; Penn et al. 2003a) are shown in Figures 3 and 4. In the presence of a radial plasma outflow constant across the penumbra, each spectral line should show a simple sinusoidal change in wavelength as a function of position angle. Rather than this simple sinusoid, the molecular lines show dark absorption across a range of wavelengths, which suggests that a range of outflow speeds are present. Higher resolution studies using other spectral lines have shown spatial variations of the flow speed, which would suggest that the large bins used here would contain a distribution of speeds (Title et al. 1993; Stanchfield, Thomas, \& Lites 1997). In both lines the absorption is consistent with typical horizontal outflow speeds of $6 \mathrm{~km} \mathrm{~s}^{-1}$ and up to roughly $9 \mathrm{~km} \mathrm{~s}^{-1}$. A brief analysis of $1565 \mathrm{~nm}$ observations of NOAA AR 9887 and NOAA AR 9888 from 2002 April 1 show outflow speeds consistent with these values.

The $\mathrm{Ti}$ I absorption line at $2231 \mathrm{~nm}$ has been used as a diagnostic of sunspot magnetic fields, since it has a large sensitivity to magnetic fields via the Zeeman effect and is thought to form predominantly in cool plasma (Ruedi et al. 1998). Magnetically sensitive lines of $\mathrm{Fe}$ I have been used with models of the solar atmospheres to investigate the Evershed outflow (Solanki, Montavon, \& Livingston 1994; Westendorp Plaza et al. 1997; Ruedi, Solanki, \& Keller 1999), but generally, spectral lines that are not sensitive to the Zeeman effect are preferred to measure the flow velocity (Wiehr \& Degenhardt 1992, 1994; Wiehr 1995, 1996). The same binning method was used with the Ti I absorption line Stokes $V$ spectra, and the results were consistent with a $6 \mathrm{~km} \mathrm{~s}^{-1}$ Evershed outflow with mean magnetic fields of $1400 \mathrm{G}$.

\section{DISCUSSION}

The CN molecular line at $1564.6 \mathrm{~nm}$ is calculated to be formed near the continuum at this wavelength at about $z=-30 \mathrm{~km}$ (defined by optical depth unity at $500 \mathrm{~nm}$ ) with a height range of about $\pm 30 \mathrm{~km}$ (H. Uitenbroek 2003, private communication).

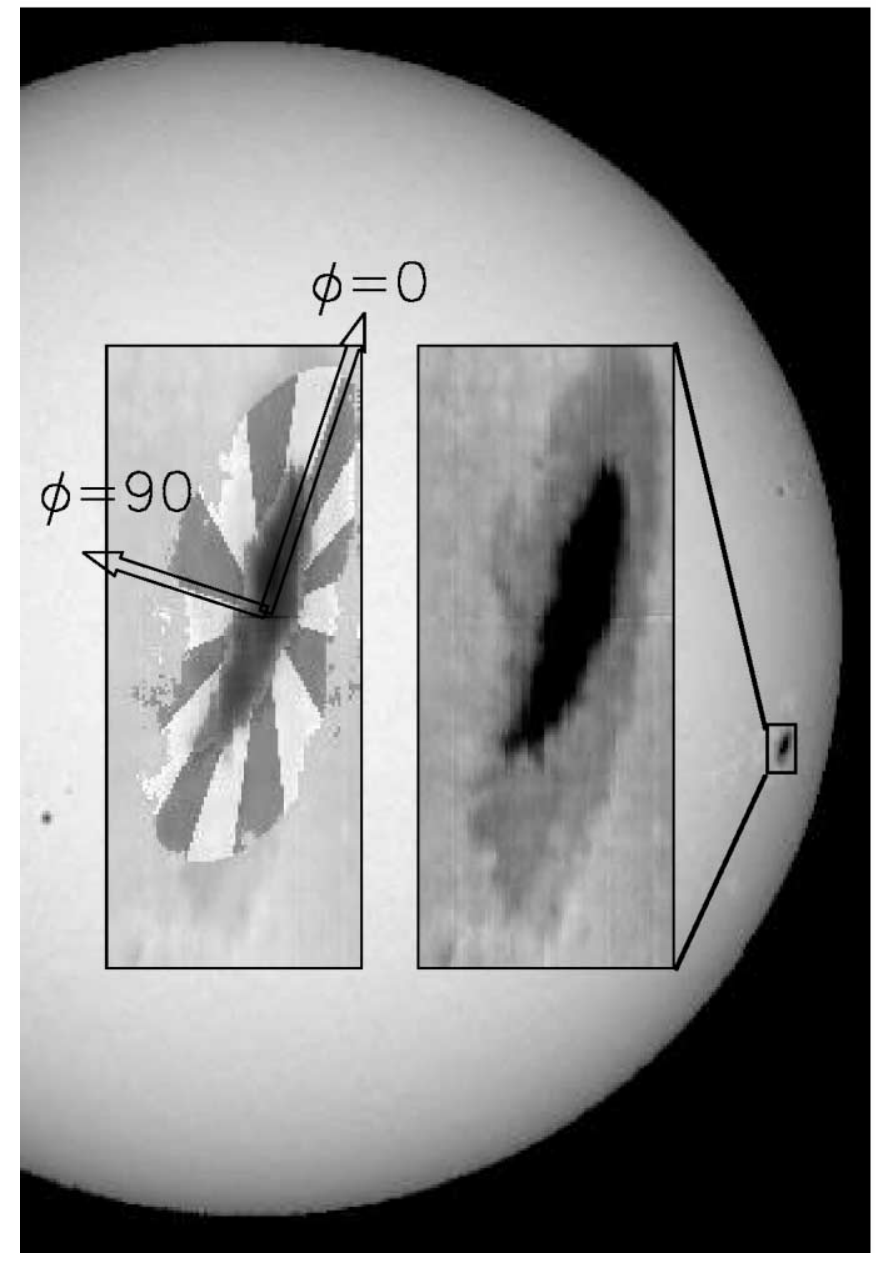

FIG. 2.-Background image from the NSO Kitt Peak Vacuum Telescope at $869 \mathrm{~nm}$ showing the disk position of NOAA AR 10008 on 2002 June 29. The two inset images show continuum maps at $2231 \mathrm{~nm}$ of the spot region, with the penumbral bins and spot azimuth directions shown in the left inset. The penumbral spectra were binned in 16 azimuthal bins, and $90^{\circ}$ azimuth is defined as toward the center of the solar disk.

Since the $2232.2 \mathrm{~nm}$ absorption line is also very weak, it is expected to be formed near the continuum at that wavelength, corresponding to a height of about $z=0 \mathrm{~km}$. While these formation heights are the deepest reported probes for the Evershed velocity, it is still instructive to compare the velocities derived here with other studies. High spatial resolution continuum images have been used to track the apparent motion of features within dark penumbral fibrils, and these measure outflow speeds of between 5.5 and $6.5 \mathrm{~km} \mathrm{~s}^{-1}$ at $z=0 \mathrm{~km}$ (Shine et al. 1994). Images taken in the wings of spectral lines and then combined to form Dopplergrams show flow speeds of about 3.5-4.7 $\mathrm{km} \mathrm{s}^{-1}$ (Rimmele 1994; Schlichenmaier \& Schmidt 2000) at heights of about $150 \mathrm{~km}$. Recent spectral frames with excellent spatial resolution (better than $350 \mathrm{~km}$ on the Sun) show asymmetric line profiles, which when fitted with two symmetric line profiles imply outflow speeds of between 5 and $7 \mathrm{~km} \mathrm{~s}^{-1}$ at heights of a few hundred kilometers (Wiehr 1995). Finally, the observations of the vertical component of the Evershed flow measure $0.2 \mathrm{~km} \mathrm{~s}^{-1}$ velocity and constrain the flow geometry to be in fibrils $20,000 \mathrm{~km}$ in length and about $300 \mathrm{~km}$ high (Rimmele 1995b). While those observations suggest changes in the inclination angle within the penumbra (which is also consistent with the observed molecular speed distribution), if 

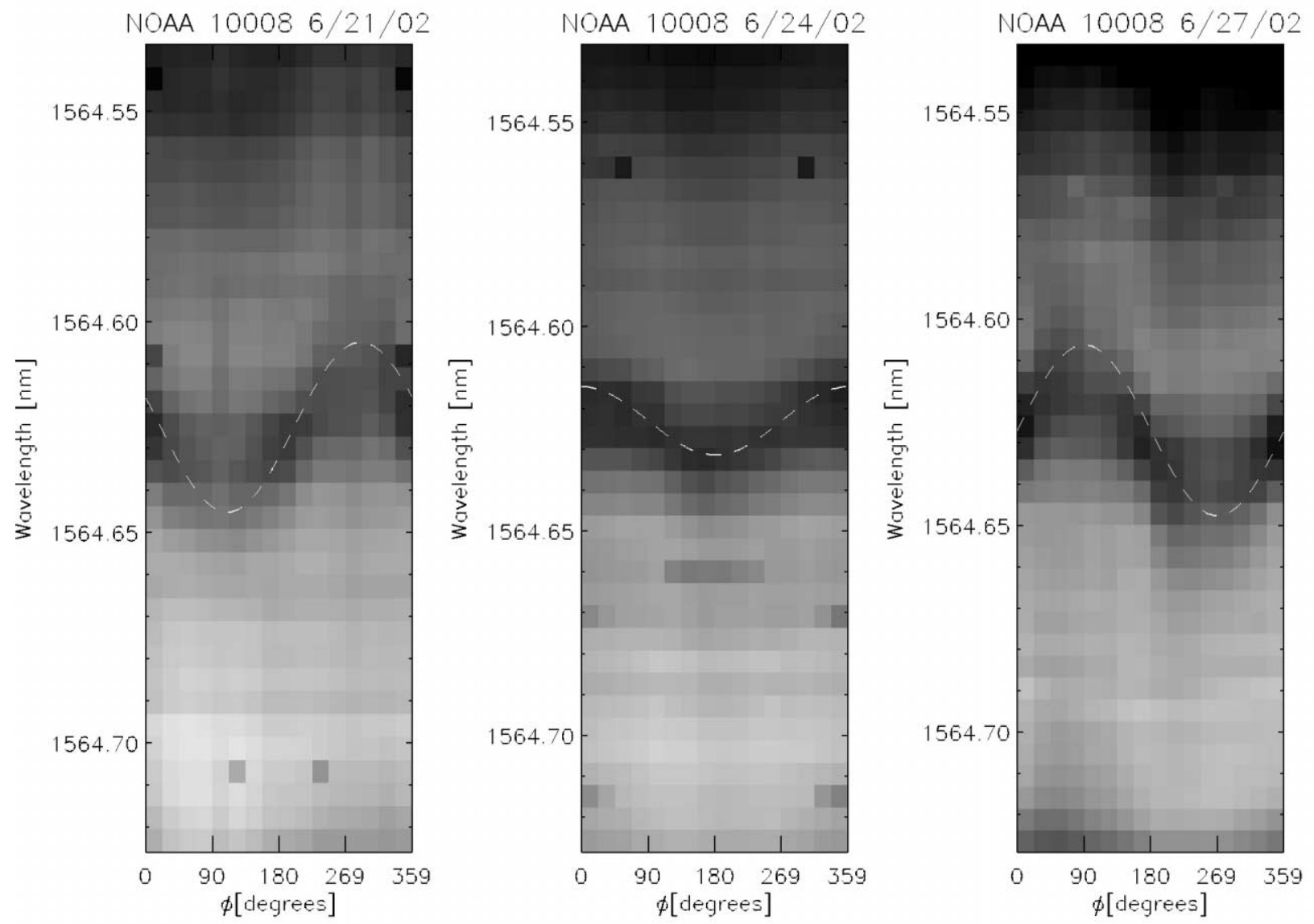

FIG. 3.-CN line at $1564.625 \mathrm{~nm}$ showing a Doppler shift as a function of azimuth angle around the sunspot NOAA AR 10008 during 3 days. The spot positions were 0.65 (east), 0.27 , and 0.66 (west) solar radii from disk center on June 21, 24, and 27, respectively. The line absorption can be seen at a variety of wavelengths ranging from zero outflow speed up to about $9 \mathrm{~km} \mathrm{~s}^{-1}$; the dashed lines show the typical horizontal speed of $6 \mathrm{~km} \mathrm{~s}^{-1}$ corrected for projection effects.

the flow is confined to a channel with an inclination of $88^{\circ} .3$ from solar vertical, then those observations imply a horizontal component of $7 \mathrm{~km} \mathrm{~s}^{-1}$.

Early work suggested that flow speed decreases with height, either linearly as $V(h)=2.5+(2.5 / 550) h$ measured by line core Doppler shifts or exponentially as $V(h)=4.5 e^{-h / 120}$ as measured by line asymmetries (Stellmacher \& Wiehr 1980). Using a depth of $-30 \mathrm{~km}$, the linear function predicts a speed of only $2.6 \mathrm{~km} \mathrm{~s}^{-1}$, while the exponential function predicts a

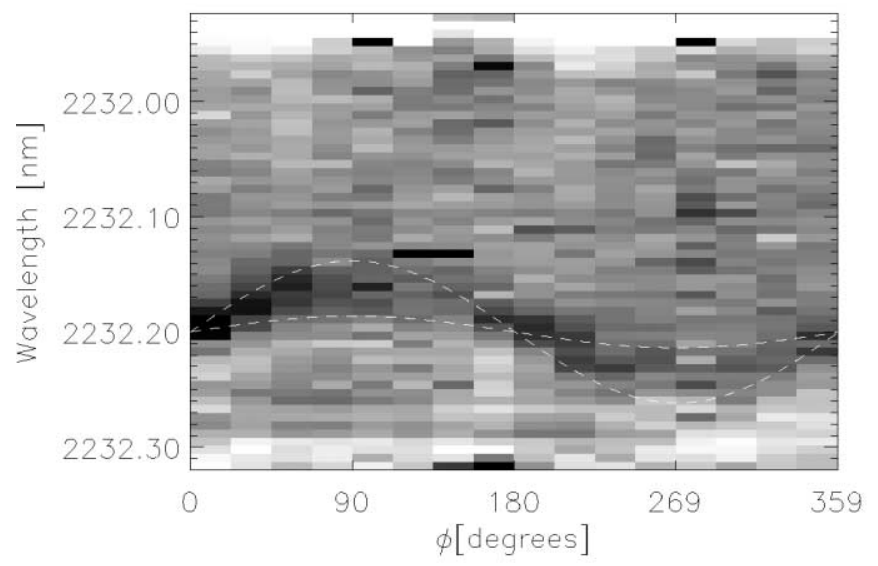

FIG. 4.- - Very weak unidentified line at $2232.2 \mathrm{~nm}$ (which shows a temperature dependence similar to $\mathrm{CN}$ ) showing Doppler shifts consistent with horizontal plasma outflows between 2 and $9 \mathrm{~km} \mathrm{~s}^{-1}$ (as shown by the dashed lines). speed of $5.8 \mathrm{~km} \mathrm{~s}^{-1}$, closer to the observed typical speed. The cause of this observed increasing speed with photospheric depth is proposed to be due to a larger effective fill factor (Degenhardt 1993). Since the molecular abundance is a strong function of plasma temperature, the effective fill factor of these lines is thought to be 1.0, and so these observations support the fill factor argument.

An early theory advanced to explain the Evershed flow was the concept of a magnetic siphon flow; a magnetic loop experiences a larger magnetic pressure in the quiet Sun than it does in the sunspot penumbra, reducing the loop's gas pressure in the quiet Sun and thus siphoning plasma out of the penumbra (Meyer \& Schmidt 1968; Thomas 1988; Degenhardt 1989; Thomas \& Montesinos 1993; Montesinos \& Thomas 1997; Thomas et al. 2002). Magnetic siphon flow theories concerning the Evershed flow have achieved success in describing the heights of the magnetic loops carrying the plasma and the thermodynamic properties of the plasma within these loops (Montesinos \& Thomas 1997; Rimmele 1995a).

While these molecular line observations are not in direct conflict with the siphon flow models, work is needed to explain the observations of these rapid flows over such large regions of the sunspot penumbra. Future models with more realistic magnetic morphology and interactions between the magnetic fields and quiet Sun may prove essential (Thomas et al. 2002).

The molecular line observations discussed here provide the most straightforward spectroscopic window on the Evershed effect in sunspots, but they are challenging observations to 
make. In this respect, two efforts are being made by the NSO to obtain higher quality infrared observations of the Sun. First, the NSO is developing a new large-format infrared camera with higher quantum efficiency and lower background levels, and it is likely to achieve first light observations in late 2003. More importantly, however, the NSO is developing plans to construct the Advanced Technology Solar Telescope (ATST), a low scattered light infrared optimized $4 \mathrm{~m}$ diameter telescope with adaptive optics. Data from the ATST promise to revolutionize our ability to make high-resolution spectroscopic observations with low photon noise. Further spectroscopic work to identify the molecular species responsible for the $2232.2 \mathrm{~nm}$ absorption line will aid future infrared Evershed observations.
The high-order adaptive optics system used at the NSO Dunn Solar Telescope to produce the image in Figure 1 was made possible through NSF award AST 00-79482. M. J. P. acknowledges support from the Air Force Office of Scientific Research through award F49620-00-1-0355 and support from NSF through ATM-0077624 and thanks T. Rimmele for a thorough review and helpful discussions. W. D. C. gratefully acknowledges the support of the K. C. Wong Education Foundation, Hong Kong, and the National Natural Science Foundation of China through award 10103004 and NSF of Yunnan through 2001A0027Q during his visit to the NSO in Tucson. The NSO/ Kitt Peak data used here are produced cooperatively by NSF/ NOAO, NASA/GSFC, and NOAA/SEL.

\section{REFERENCES}

Beckers, J. M., \& Schroter, E. H. 1969, Sol. Phys., 10, 384

Bhatnagar, A., Livingston, W. C., \& Harvey, J. W. 1972, Sol. Phys., 27, 61

Degenhardt, D. 1989, A\&A, 222, 297 . 1993, A\&A, 277, 235

del Toro Iniesta, J. C., Bellot Rubio, L. R., \& Collados, M. 2001, ApJ, 549, L139

Ding, M. D., \& Fang, C. 1989, A\&A, 225, 204

Evershed, J. 1909, MNRAS, 69, 454

Meyer, F., \& Schmidt, H. U. 1968, Z. Angew. Math. Mech., 48, 218

Moe, O. K., \& Maltby, P. 1974, Sol. Phys., 36, 101

Montesinos, B., \& Thomas, J. H. 1997, Nature, 390, 485

Penn, M. J., Cao, W. D., Walton, S. R., Chapman, G., \& Livingston, W. 2003a, Sol. Phys., 213, 55

Penn, M. J., \& LaBonte, B. J. 1993, ApJ, 415, 383

Penn, M. J., Walton, S., Chapman, G., Ceja, J., \& Plick, W. 2003b, Sol. Phys., in press

Rimmele, T. 1994, A\&A, 290, 972 1995a, A\&A, 298, 260 1995b, ApJ, 445, 511

Ruedi, I., Solanki, S. K., \& Keller, C. U. 1999, A\&A, 348, L37

Ruedi, I., Solanki, S. K., Keller, C. U., \& Frutiger, C. 1998, A\&A, 338, 1089
Scharmer, G. B., Gudiksen, B. V., Kiselman, D., Lofdahl, M. G., \& Rouppe van der Voort, L. H. M. 2002, Nature, 420, 151

Schlichenmaier, R., \& Schmidt, W. 2000, A\&A, 358, 1122

Shine, R. A., Title, A. M., Tarbell, T. D., Smith, K., Frank, Z. A., \& Scharmer, G. 1994, ApJ, 430, 413

Solanki, S. K., Montavon, C. A. P., \& Livingston, W. 1994, A\&A, 283, 221

Stanchfield, D. C. H., Thomas, J. H., \& Lites, B. W. 1997, ApJ, 477, 485

Stellmacher, G., \& Wiehr, E. 1980, A\&A, 82, 157

Thomas, J. H. 1988, ApJ, 333, 407

Thomas, J. H., \& Montesinos, B. 1993, ApJ, 407, 398

Thomas, J. H., Weiss, N. O., Tobias, S. M., \& Brummell, N. H. 2002, Nature, 420, 390

Title, A. M., Frank, Z. A., Shine, R. A., Tarbell, T. D., Topka, K., \& Scharmer, G. 1993, ApJ, 403, 780

Westendorp Plaza, C., del Toro Iniesta, J. C., Ruiz Cobo, B., \& Martinez Pillet, V. 2001, ApJ, 547, 1148

Westendorp Plaza, C., del Toro Iniesta, J. C., Ruiz Cobo, B., Martinez Pillet, V., Lites, B. W., \& Skumanich, A. 1997, Nature, 389, 47

Wiehr, E. 1995, A\&A, 298, L17 1996, A\&A, 309, L4

Wiehr, E., \& Degenhardt, D. 1992, A\&A, 259, 313 . 1994, A\&A, 287, 625 